\title{
'Neural histoid'. Histoid leproma in peripheral nerve; a case report.
}

\author{
K RAMANUJAM, S ARUNTHATHI, C J G CHACKO \\ \& MARY JACOB \\ Schieffelin Leprosy Research \& Training Centre (SLR \& TC) \\ Karigiri, N. Arcot District, Tamil Nadu, 632106 India
}

Accepted for publication 6 September 1983

\begin{abstract}
Summary A case of 'neural histoid' nodules occurring in the peripheral and cutaneous nerves, in an otherwise inactive case of lepromatous leprosy is presented. It is postulated that this is an instance of relapse of the disease occurring exclusively in the nerve, the nodules resulting from the unrestricted multiplication of drug-resistant mutants. The pathogenesis of the condition is discussed.
\end{abstract}

\section{Introduction}

In 1963 Wade described a variant of the conventional nodular lepromatous leprosy to which he gave the name 'Histoid' variety of lepromatous leprosy.' Clinically he identified three types of lesions - the 'cutaneous' and the 'subcutaneous' histoids occurring in the cutis and the subcutis; and the 'sharply delimited plaques, on the points of pressure'. Several publications that followed confirmed Wade's observations regarding the occurrence of these lesions and in the locations mentioned by him.

A lepromatous case who had been inactive for over 9 years and on continuous dapsone monotherapy was referred from the Department of Epidemiology, SLR $\&$ TC, for an opinion regarding the small lumps that had arisen over the radial cutaneous nerves. Clinically the lumps were suggestive of nerve abscesses. One of the lumps on histopathological examination proved to be a histoid nodule in the nerve. In view of this interesting finding and the paucity of reports on histoid lesions in the nerve, the details of the case along with the investigations and findings are presented here.

\section{Case report}

Patient S, male, aged 27 years, was admitted at the SLR \& TC in January 1969 as a 


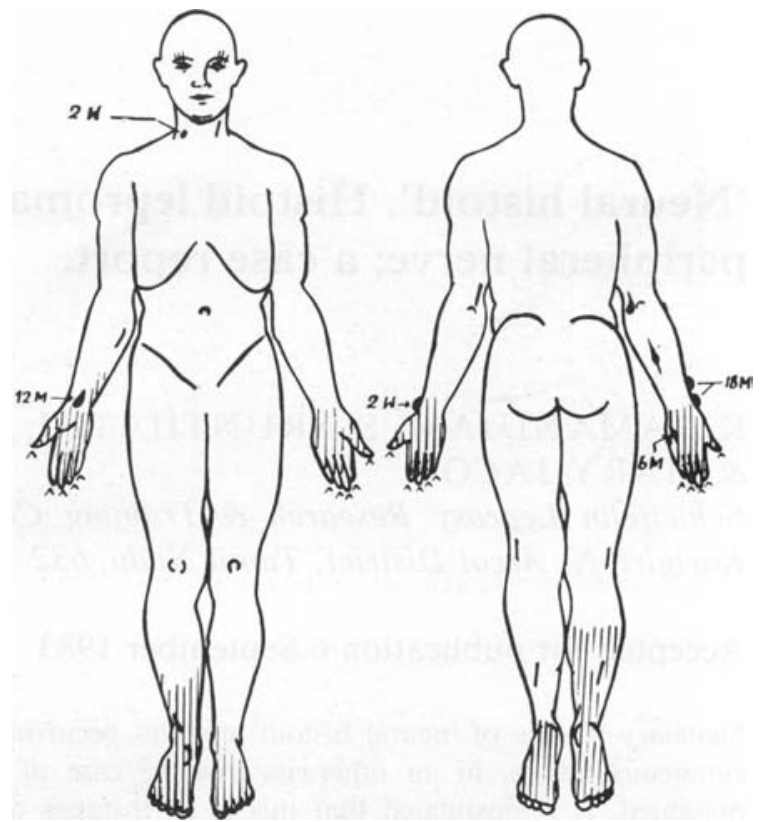

Figure 1. Chart showing the clinical condition of the patient, 7 October 1982.

lepromatous case with recurrent reactive episodes. His BI was 3.75 . Initially he was treated with antimalarials and antimonials and later with clofazimine. Dapsone therapy was then started, reaching up to $100 \mathrm{mg}$ per day. Attacks of neuritis continued to occur. The dose of dapsone was reduced to $50 \mathrm{mg}$ per day and continued regularly. He became bacteriologically negative in May 1973. Subsequently, he developed neuritis on two occasions. In course of time ENL and neuritis abated. Dapsone was continued. The skin smears remained negative.

In October 1982, he was referred to us for an opinion of the small swellings over the radial cutaneous nerves. Clinical examination showed that he was an inactive case of lepromatous leprosy with marked thickening of several peripheral and cutaneous nerves and anaesthesia over the hands and feet (Figure 1). The nerves were firm and non-tender with nodular swellings over some of them (Figure 2). Some of the nodules were of 6-18 months' duration and the others had been present for 2 weeks. The latter were erythematous and tender. There was a suggestion of fluctuation over one of the swellings. A tentative diagnosis of '?nerve abscess' was made and the case was referred to a surgeon for exploration and biopsy of one of the nodules. Skin smears were negative.

The first biopsy was taken from the pearly nodule over the thickened right posterior antebrachial nerve and sent for histopathological examination with a clinical diagnosis of '?neuroma ?histoid nodule'. The report came as 'histoid nodule, nerve'. This unexpected finding stimulated further interest in the case and 


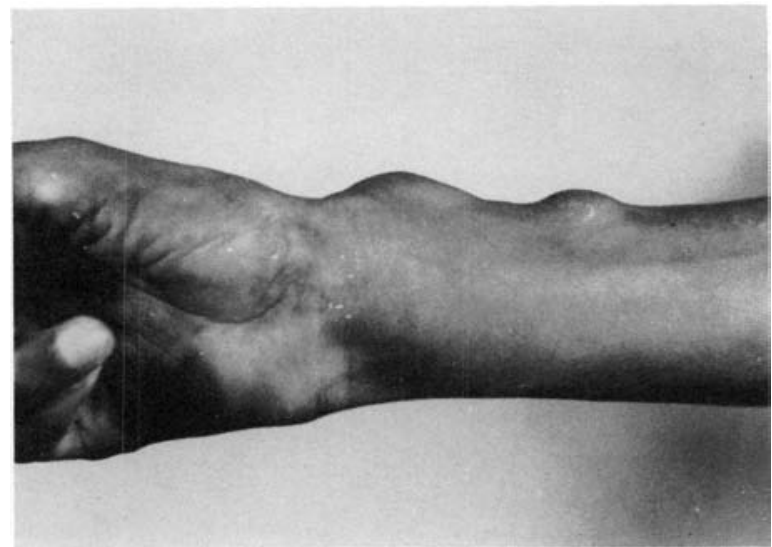

Figure 2. Large swellings in the region of the right wrist.

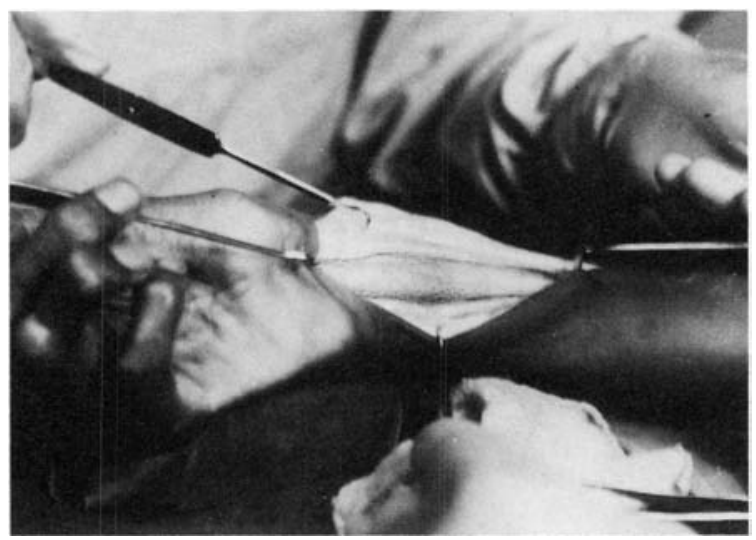

Figure 3. Exploration of the median at the wrist revealing the large fusif orm swelling in the nerve prior to its entry into the carpal tunnel.

led to the exploration, biopsy and histological examination of the nodule over the right radial cutaneous and the large, firm, fusiform swelling over the right median at the wrist (Figure 3). A biopsy from the skin over the left back was also taken. A piece from the nodule was also sent for mouse foot-pad inoculation on the suspicion that this could be a manifestation of relapse in the nerve by drug-resistant mutants. The patient commenced multidrug therapy.

The contact skin smears from the cut surface of the nodules showed innumerable bacilli, many of them solid. Histopathologically, all three biopsies from the nodular swelling in the nerves showed nerve bundles partly or completely replaced by spindle-shaped cells showing a whorled arrangement (Figures 4 and 5). Another area showed tissue breakdown with many red cells and occasional polymorph. Moderate number of lymphocytes were seen at the 


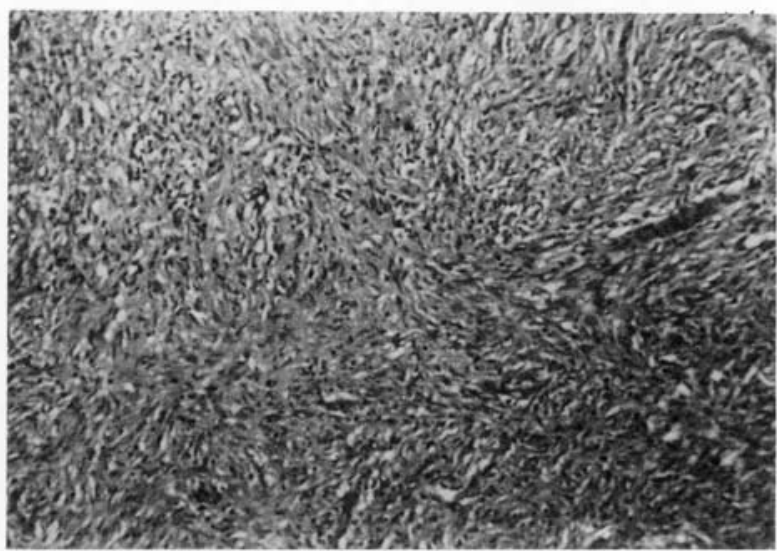

Figure 4. Spindle cells arranged in a whorled pattern, characteristic of histoid leprosy.

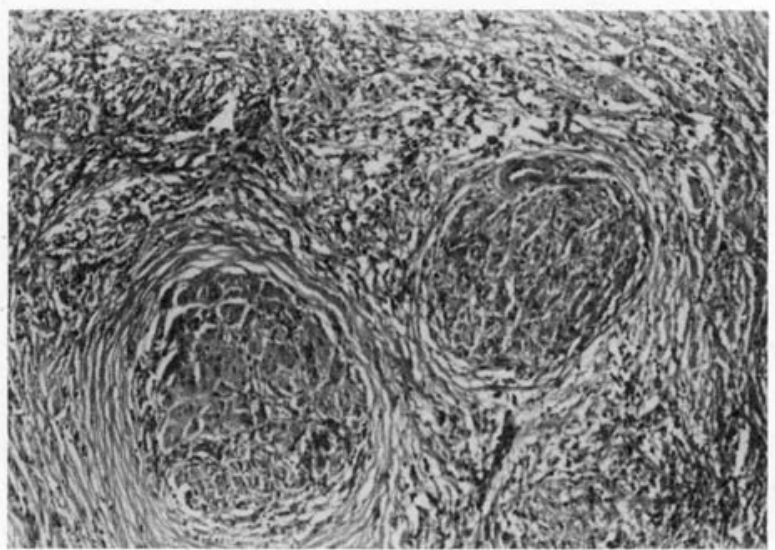

Figure 5. Two fasicles of nerves with perineural lamination and spindle cells with lymphocytes in the adjacent tissue.

periphery of some lesions. Acid-fast stain showed clumps of granular and solid bacilli. The biopsy from the residual skin lesion over the back revealed a picture consistent with resolved leprosy.

\section{Discussion}

Histoid lepromatous leprosy manifesting with nodules and plaques in the skin and subcutis have been documented by several workers. The occurrence of such lesions in situations other than those mentioned by Wade have not been recorded except in one instance. ${ }^{9}$ Wade, however, observed that 'until detailed case reports 
can be presented, little can be said about the clinical variations of the cases presenting with histoid lesions'. Perhaps, he considered that the histoid lesions could occur in other situations as well.

Regarding the genesis of histoid lesions, Wade said that such cases arose in long-standing lepromatous cases who under treatment registered varying grades of improvement, even to the point of negativity in the skin smears, 'but later reactivation or relapse occurred'. 'As far back as 1963, while on a visit to India, it was demonstrated to him, that histoid lesions can occur, per se, in virgin lepromatous cases. ${ }^{3}$

Some workers consider that histoid lesions can arise as an expression of the rapid multiplication of drug-resistant mutants in the multibacillary cases who had been on antileprosy treatment and apparently recovered..$^{2,4}$.

It has been postulated ${ }^{5}$ that in addition to the general concept that histoid lesions arose as a result of focalized rapid multiplication of drug-resistant Mycobacterium leprae, these lesions were essentially an expression of 'an alteration in the growth pattern of the organism in a localized area of skin', and the change in the behaviour of the organism may be due to "loss of immunity in the localized area of skin', resulting in the enhancement of the bacillary multiplication.

It is stated ${ }^{6}$ in an ultrastructural observation of histoid leprosy that 'the histoid lesion is an hyperactive form of lepromatous leprosy, the nature of the stimulus being unknown'. The opinion given is that 'although the histoid lesions are found most commonly in relapsing patients, whether or not the relapse is due to drug resistance, this is not necessarily the case and the stimulus that produces the high cell-turnover has not been elucidated'.

From the foregoing it would appear that the occurrence of histoid lesions could be the outcome of unchecked, progressive lepromatous leprosy or of relapse, the former arising from the unrestricted multiplication of virgin $M$. leprae not exposed to any antileprosy treatment and the latter from drug-resistant bacilli. Apart from these, there is yet another source from where such a phenomenon could occur. These are the nests of 'persisters' which have been identified in no less than five sequestered locations in the multibacillary cases, long after the bacilli had cleared from the skin and the nasal mucosa as determined by standard methods of bacteriological examination. One of the sites of persistence is the Schwann cell. It is said that some of the persisters die in the course of time while others regain normal metabolic activity and multiply. ${ }^{7}$ It is not improbable that these persisters in the Schwann cells come out of their hiding and hibernation, become metabolically active, multiply at a prolific rate, producing histoid type of lesions.

It is postulated that the occurrence of firm, non-tender nodular elements in the peripheral and cutaneous nerves in the case under report is a unique instance of relapse occurring exclusively in the nerve, the skin remaining apparently free from relapse lesions; and that the nodules arose as the result of unrestricted 
multiplication of drug-resistant bacilli. The results of foot-pad inoculation showed that the organisms were fully resistant to dapsone.

In this context it is of interest to refer to two publications ${ }^{8,9}$ reporting on the occurrence of histoid features in the nerve. The presence of histoid features were found in the histological examination of a swelling in the radial cutaneous nerve in a case of histoid lepromatous leprosy, which was mistaken for a nerve abscess. The presence of spindle-shaped cells were observed ${ }^{9}$ with many full-stained bacilli in the histological examination of one of the 'large subcutaneous nodules which were attached to the nerve trunks in an untreated lepromatous case'.

This case report on the occurrence of 'neural histoid', though it may now appear to be a clinicopathological curiosity or rarity, it is not unlikely that its frequency can be much more in the present context when drug resistance and microbial persistence continue to be the two serious problems in the therapeutic management of multibacillary leprosy. Perhaps, one may be inclined to pass off these swellings as 'nerve abscess', as almost happened in this case. More cases will be discovered if clinicians are aware of this clinical entity in relapsed as well as active progressive, unchecked lepromatous cases manifesting with histoid features, supported by facilities of histopathological examination.

\section{Acknowledgements}

We thank the Department of Epidemiology, SLR \& TC, for referring the case, Drs E P Fritschi and S Ramaprasad for exploration and biopsy of the swellings Sri Ramadoss for the clinical photographs, Sri J Ramamurthy for secretarial assistance and patient $\mathrm{S}$ for his most willing co-operation.

\section{References}

1 Wade HW. The histoid variety of lepromatous leprosy. Int J Lepr, 1963; 31: 129-42.

2 Rodriguez JN. The histoid leproma. Its characteristics and significance. Int J Lepr, 1969; 37: 1-21.

3 Ramanujam K, Ramu G. Wade's histoid lepromatous leprosy. Lepr India, 1969; 41: 293-7.

${ }^{4}$ Desikan KV, Iyer CGS. Histoid variety of lepromatous leprosy. A histological study. Int J Lepr, 1972; 40: 149-56.

5 Job CK, Chacko CJG, Taylor PM. Electronmicroscopic study of histoid leprosy with special reference to its histogenesis. Lepr India, 1977; 49: 467-70.

${ }^{6}$ Ridley MJ, Ridley DS. Histoid leprosy. An ultrastructural observation. Int J Lepr, 1980; 48: 135-9.

7 Kurt Toman. Bacterial persistence in leprosy. Int J Lepr, 1981; 49: 205-17.

${ }^{8}$ Roy Choudhury SB, Srinivasan H. Nerve abscess in lepromatous leprosy. A case report and discussion of pathogenesis. Lepr India, 1977; 49: 330-8.

9 Gaulier A. Spindle shaped leproma cells arising from perineurium. A link with Wade's histoid leprosy, one case. Arch Anat Cytol Path, 1980; 28: 277-82. (In French) Int J Lepr, 1981; 49: 476. 